\title{
The Study of Heavy Vehicles Rollover Warning Method Based on the Suspension Distortion Perception
}

\author{
Zhiguo Zhao ${ }^{*}, 1,2$, Min Chen ${ }^{2}$ and Chao Wang ${ }^{1}$ \\ ${ }^{1}$ School of Transportation Engineering, Huaiyin Institute of Technology, Huai'an, 223001, China \\ ${ }^{2}$ School of Mechanical Engineering, Southeast University, Nanjing, 211189, China
}

\begin{abstract}
With the features of high centroid, high load capacity and high aspect ratio, heavy vehicles are prone to rollover when running at high speeds in the corners, so the study of heavy vehicle rollover warning method presents important realistic meanings. In this paper, firstly, the composition of early warning systems is analyzed, and then the principles of rollover warning method based on suspension distortion perception are proposed; Secondly, the wheels sprung suspension force formula is established when the vehicle is cornering, taking lateral load transfer rate as the rollover warning threshold and the size of wheel suspension pressure warning threshold when cornering is determined; Finally, a test vehicle is taken for example to validate the rollover warning method. The simulation results show that the early warning method is capable of warning the vehicles when in the curve and has a rollover risk to avoid the rollover, thus improving the traffic efficiency in curve.
\end{abstract}

Keywords: Heavy vehicle, Rollover, Suspension distortion, Suspension load, Warning method.

\section{INTRODUCTION}

Highway curve is the black-spot of accident; the probability of the accident to occur and the high-severity of the accident have made it a concerning issue in research institutes and universities domestically and abroad [1,2]. National Highway Traffic Safety Administration statistics show that the degree of hazard of vehicles rollover accident is just secondary to the car crash accident. According to the University of Michigan Transportation Research Center statistics, during the period 2002-2006, heavy vehicles rollover accidents occurred 5200 times annually on an average in the US (average deaths amounting to 5,300). Furthermore, the deaths due to heavy vehicles rollover accidents are increasing year by year [3], being times more in China than European and American countries. Visibly, heavy vehicle rollover accidents are the major traffic accidents that cause the loss of life and property and have become important issues affecting transportation safety.

In recent years, scholars in China and abroad have conducted much relevant research on rollover warning of heavy vehicles traveling on a curve. Rollover warning methods mainly consist of rollover warning time TTR (time to rollover) $[4,5]$, rollover risk prediction [6] and use of lateral acceleration and the lateral load transfer rate as the early warning indicators to determine the degree of risk of vehicle rollover warning $[7,8]$ and so on. Although scholars have carried out a lot of research on vehicle rollover warning method, but with the view to improve the accuracy and timeliness of rollover warning, there is still a lot of work to be done through further exploration and research.

*Address correspondence to this author at the School of Transportation Engineering, Huaiyin Institute of Technology, 1 East Meicheng Road, Huaiyan, Postcard: 223003, China; Tel: +86+517-83559165;

E-mail: 51066347@qq.com
In this paper, the rollover warning system of vehicles running in curve is analyzed; a rollover warning method is proposed based on suspension distortion perception which takes lateral load transfer ratio as vehicle rollover evaluation index. When index of one of the wheels reaches the threshold calculated by warning system, the system can timely warn and alert the driver to control the vehicle to prevent rollover.

\section{ROLLOVER WARNING SYSTEM AND THE PRINCIPLE}

\subsection{Composition of Rollover Warning System}

Rollover warning system is composed of the suspension system, the data acquisition module and the vehicle mounted device. Leaf spring is the buffer unit in the suspension system; there are leaf spring displacement sensors, lateral acceleration sensor, and cross slope sensor in the data acquisition module to monitor vehicle operating status timely; the vehicle mounted device includes processor module, data receiving module, alarm module, etc.

\subsection{Principle of Rollover Warning System}

As the vertical load on wheels of the vehicle constantly changes, which is difficult to measure, so this paper took displacement of the leaf spring in the process of compression as measured parameter and used displacement sensors to monitor compression displacement for warning [9]. The pressure is equal to the product of the spring rate and the amount of displacement, making pressure threshold values equivalent to the displacement threshold which are $\mathrm{H}_{\mathrm{Lmin}}$, $\mathrm{H}_{\text {Rmax }}, \mathrm{H}_{\text {Lmax }}, \mathrm{H}_{\text {Rmin }}$.

Fig. (1) shows the process of principle of rollover warning. 


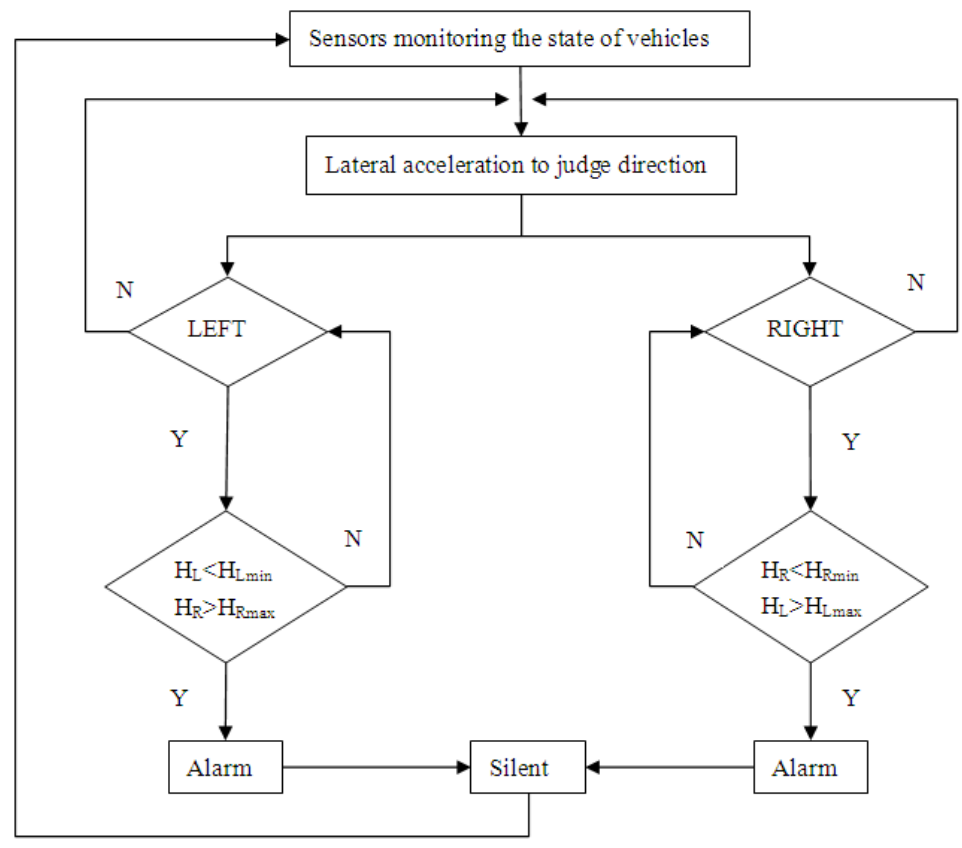

Fig. (1). The process of principle of rollover warning.

Rollover warning proceeds as follows:

a) When driving, the vehicle develops sensor operation real-time monitoring. The lateral acceleration sensor directs the vehicle when to turn and dictates its steering direction; the transverse cross slope sensor monitors the slope when the vehicle is traveling; the displacement sensor of the leaf spring detects the changes of compression displacement and amount of the leaf spring of suspension system to characterize the change of wheel loads.

b) When the vehicle steers, the steering direction is judged by monitoring data from the lateral acceleration sensor, the system transfers the various parameters monitored to the processor means for calculating the real-time warning threshold of compression of the vehicle wheel suspension, and then compares with the current actual amount of displacement, and determines whether there is a danger of rollover.

c) If there is risk of rollover, the alarm unit sends an alarm signal to the driver and alerts the driver to take real-time measures to reduce the speed or decrease the steering angle of rotation to avoid vehicle rollover.

d) If there is no risk of rollover, the system stays silent and re-calculates warning thresholds in real time for comparing again.

e) The system remains silent until the vehicle steering is completed.

\section{ROLLOVER WARNING THRESHOLD}

\subsection{Steering Wheel Suspension Sprung Pressure when Turning Left}

Fig. (2) shows the schematic diagram of force of the front suspension spring force when turning left; the parameters in Fig.
(1) have the following meanings: $M_{I F}$ is the front axle sprung mass, $M_{I B}$ is the rear axle sprung mass, $N_{R F}$ is the sprung pressure value of outside of the right front wheel when turning left, $N_{L F}$ is the sprung pressure value of outside of the left front wheel when turning right, $a_{y F}$ is the front axle lateral acceleration, $a$ is cross slope angle, $B_{F}$ is the left-right spring spacing of front suspension, $B_{B}$ is the left-right spring spacing of rear suspension, $h_{F}$ is the vertical distance form front suspension sprung mass centroid height to the front axle, $h_{B}$ is the vertical distance form rear suspension sprung mass centroid height to the rear axle and $g$ is the acceleration of gravity.

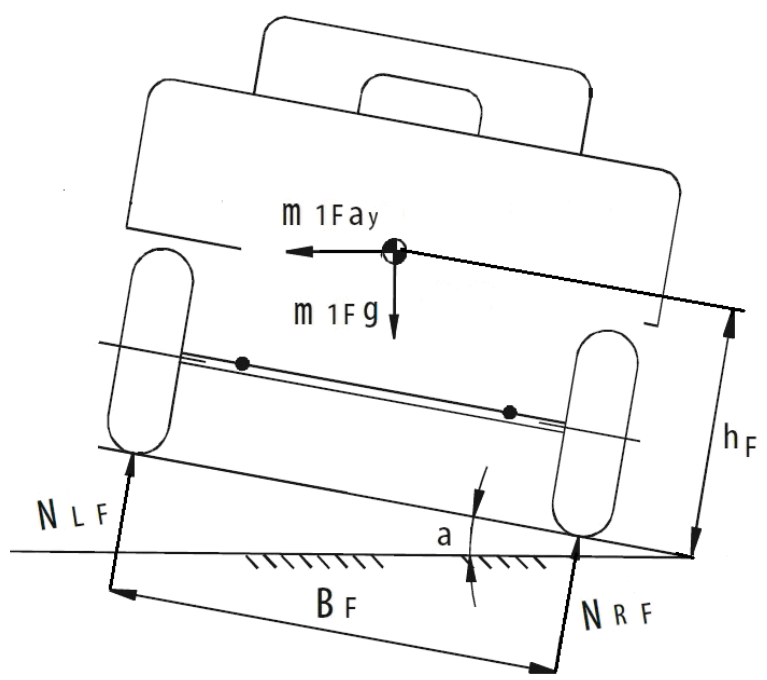

Fig. (2). The schematic diagram of force of front suspension spring force when turning left.

From Torque balance, we get:

$$
\begin{aligned}
& N_{L F} \cdot B_{F}-h_{F} \cdot\left(M_{1 F} \cdot a_{y F} \cdot \cos \alpha-M_{1 F} \cdot g \cdot \sin \alpha\right) \\
& =\frac{B_{F}}{2} \cdot M_{1 F} \cdot a_{y F} \cdot \sin \alpha+M_{1 F} \cdot g \cdot \frac{B_{F}}{2} \cdot \cos \alpha
\end{aligned}
$$


So the sprung pressure value of inside of the left front wheel is:

$$
\begin{array}{r}
M_{1 F} \cdot g \cdot\left(\frac{B_{F}}{2} \cdot \cos \alpha+h_{F} \cdot \sin \alpha\right)+ \\
N_{L F}=\frac{M_{1 F} \cdot a_{y F} \cdot\left(\frac{B_{F}}{2} \cdot \sin \alpha-h_{F} \cdot \cos \alpha\right)}{B_{F}}
\end{array}
$$

Similarly, the sprung pressure value of inside of the left rear wheel is:

$$
\begin{array}{r}
M_{1 B} \cdot g \cdot\left(\frac{B_{B}}{2} \cdot \cos \alpha+h_{B} \cdot \sin \alpha\right)+ \\
N_{L B}=\frac{M_{1 B} \cdot a_{y B} \cdot\left(\frac{B_{B}}{2} \cdot \sin \alpha-h_{B} \cdot \cos \alpha\right)}{B_{B}}
\end{array}
$$

From the force balance, we can get:

$N_{R F}=M_{1 F} \cdot \mathrm{g} \cdot \cos \alpha+M_{1 F} \cdot a_{y F} \cdot \sin \alpha-N_{L F}$

So the sprung pressure value of outside of the right front wheel is:

$$
\begin{array}{r}
M_{1 F} \cdot g \cdot\left(\frac{B_{F}}{2} \cdot \cos \alpha-h_{F} \cdot \sin \alpha\right)+ \\
N_{R F}=\frac{M_{1 F} \cdot a_{y F} \cdot\left(\frac{B_{F}}{2} \cdot \sin \alpha+h_{F} \cdot \cos \alpha\right)}{B_{F}}
\end{array}
$$

Similarly, the sprung pressure value of outside of the right rear wheel is:

$$
\begin{array}{r}
M_{1 B} \cdot g \cdot\left(\frac{B_{B}}{2} \cdot \cos \alpha-h_{B} \cdot \sin \alpha\right)+ \\
N_{R B}=\frac{M_{1 B} \cdot a_{y B} \cdot\left(\frac{B_{B}}{2} \cdot \sin \alpha+h_{B} \cdot \cos \alpha\right)}{B_{B}}
\end{array}
$$

\subsection{Steering Wheel Suspension Sprung Pressure when Turning Right}

Fig. (3) is the schematic diagram of force of the front suspension spring force when turning right.

From Torque balance, we get:

$N_{R F} \cdot B_{F}-M_{1 F} \cdot a_{y F} \cdot h_{F} \cdot \cos \alpha$

$=\frac{B_{F}}{2} \cdot\left(M_{1 F} \cdot g \cdot \cos \alpha-M_{1 F} \cdot a_{y F} \cdot \sin \alpha\right)+M_{1 F} \cdot g \cdot h_{F} \cdot \sin \alpha$

So the sprung pressure value of inside of the right front wheel is:

$$
\begin{array}{r}
M_{1 F} \cdot g \cdot\left(\frac{B_{F}}{2} \cdot \cos \alpha+h_{F} \cdot \sin \alpha\right)+ \\
N_{R F}=\frac{M_{1 F} \cdot a_{y F} \cdot\left(h_{F} \cdot \cos \alpha-\frac{B_{F}}{2} \cdot \sin \alpha\right)}{B_{F}}
\end{array}
$$

Similarly, the sprung pressure value of inside of the right rear wheel is:

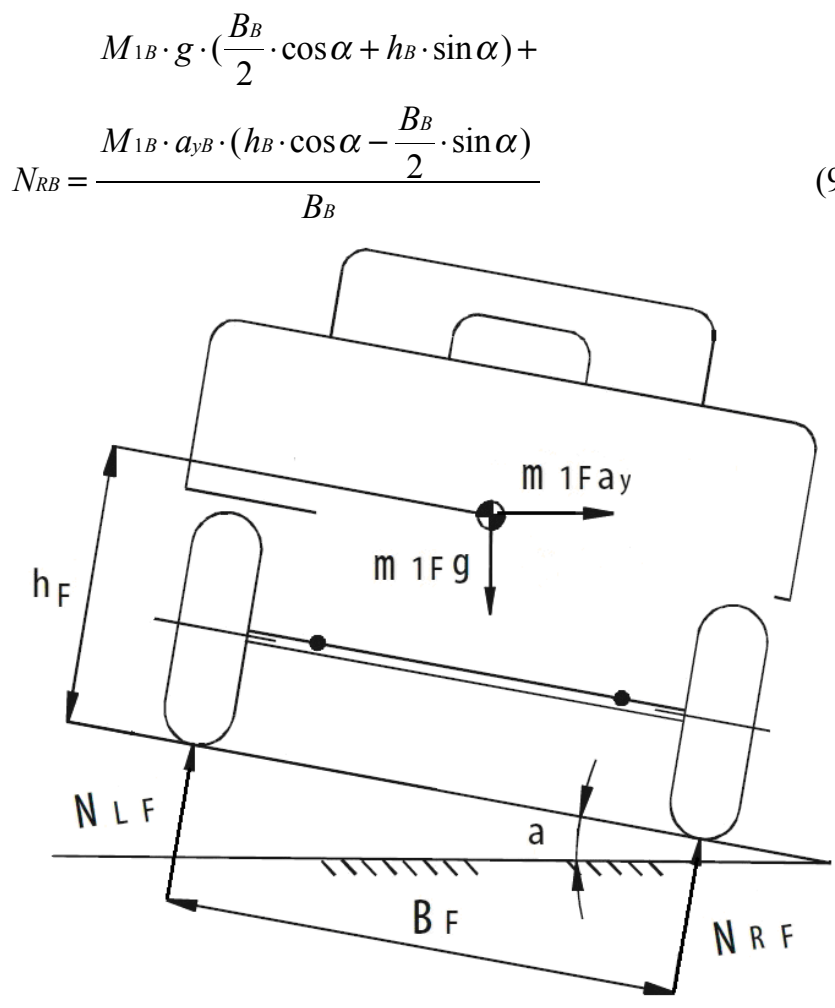

Fig. (3). The schematic diagram of force of front suspension spring force when turning right.

From force balance, we can get:

$N_{L F}=M_{1 F} \cdot \mathrm{g} \cdot \cos \alpha-M_{1 F} \cdot a_{y F} \cdot \sin \alpha-N_{R F}$

So the sprung pressure value of outside of the left front wheel is:

$M_{1 F} \cdot g \cdot\left(\frac{B_{F}}{2} \cdot \cos \alpha-h_{F} \cdot \sin \alpha\right)-$
$N_{L F}=\frac{M_{1 F} \cdot a_{y F} \cdot\left(\frac{B_{F}}{2} \cdot \sin \alpha+h_{F} \cdot \cos \alpha\right)}{B_{F}}$

Similarly, the sprung pressure value of outside of the left rear wheel is:

$$
\begin{array}{r}
M_{1 B} \cdot g \cdot\left(\frac{B_{B}}{2} \cdot \cos \alpha-h_{B} \cdot \sin \alpha\right)- \\
N_{L B}=\frac{M_{1 B} \cdot a_{y B} \cdot\left(\frac{B_{B}}{2} \cdot \sin \alpha+h_{B} \cdot \cos \alpha\right)}{B_{B}}
\end{array}
$$

\subsection{Determination of Rollover Warning Threshold}

Lateral load transfer ratio is introduced, which is defined as the ratio of the difference and the sum of wheels vertical loads [10]. This method can predict the emergency that one side of the vehicle is going off the ground according to the magnitude of displacement of the leaf spring. LTR is defined as follows:

$$
L T R=\frac{\left|N_{L}-N_{R}\right|}{N_{L}+N_{R}}
$$


In the formula above, $N_{L}-$ supports reaction of left wheel ; $N_{R}$ - supports reaction of left wheel.

Taking vehicles turning left for example, when LTR is 0.85 , steering wheel inside suspension sprung pressure when turning is warning threshold of the system. (AUTHOR: Please rephrase for clarity) According to equation (13), support reaction of left and right wheels front axle meets $\frac{N_{\mathrm{L}}}{N_{R}}=\frac{3}{37}$.

According to the proportional conditions satisfied with the left and right wheels of the front Axle support reaction force and the equation (2)and(5), warning lateral acceleration $\mathrm{a}_{\mathrm{ymax}}$ is obtained,

$a_{y \max }=\frac{17 B+40 h_{g} \cdot \tan a}{17 B \tan a-40 h_{g}} \cdot g$

Incorporating $\mathrm{a}_{\mathrm{ymax}}$ into equation (2), rollover warning threshold of pressure on the left side of the suspension $\mathrm{N}_{\mathrm{Lmin}}$ is obtained; $a$ is same so it gives rollover warning lateral acceleration of the right side suspension and the corresponding pressure threshold $\mathrm{N}_{\mathrm{Rmax}}$.

When the vehicle is turning right, rollover warning threshold of pressure of the suspensions is $\mathrm{N}_{\mathrm{Lmax}}$, and $\mathrm{N}_{\mathrm{Rmin}}$ with $a$ being similar.

\subsection{Examples of Simulation}

Taking a 35-ton truck for vehicle mounted unit, the length is $12.5 \mathrm{~m}$, the width is $3 \mathrm{~m}$, the height is $2.7 \mathrm{~m}$ and the other parameters are listed in Table $\mathbf{1}$.

Table 1. Structural parameters of truck.

\begin{tabular}{|c|c|c|c|c|}
\hline $\begin{array}{c}\text { Centroid High } \\
\boldsymbol{h}_{\mathrm{g}} / \mathbf{m}\end{array}$ & $\boldsymbol{g} /\left(\mathbf{m} / \mathbf{s}^{2}\right)$ & $\begin{array}{c}\text { Tread } \\
\boldsymbol{B} / \mathbf{m}\end{array}$ & $\begin{array}{c}\text { Mass } \\
\boldsymbol{M} / \mathbf{k g}\end{array}$ & $\begin{array}{c}\text { Spring Stiffness } \\
\boldsymbol{K} /(\mathbf{k N} / \mathbf{m m})\end{array}$ \\
\hline \hline 2 & 9.8 & 2.5 & 35000 & 1.769 \\
\hline
\end{tabular}

Selecting a junction as the experimental zone path parameter: $a$ is the inclination angle of curve 3 and $\mathrm{R}$ is the turn radius $25 \mathrm{~m}$. A case study of experimental vehicles turning left, from equation (13), the relationship between the vehicle vertical loads of wheels can be obtained as

$$
\frac{N_{\mathrm{L}}}{N_{R}}=\frac{3}{37} \text {. }
$$

By putting data that we have into equation (2) and (5), the parameters calculation results can be obtained which are shown in Table 2.

Table 2. Calculation results of threshold.

\begin{tabular}{|c|c|c|c|c|c|}
\hline $\boldsymbol{N}_{L} / \mathbf{k N}$ & $\boldsymbol{H}_{L} / \mathbf{m m}$ & $\boldsymbol{N}_{R} / \mathbf{k N}$ & $\boldsymbol{H}_{\boldsymbol{R}} / \mathbf{m m}$ & $\boldsymbol{L T R}$ & $\boldsymbol{a}_{\text {ymax }} /\left(\mathbf{m} / \mathbf{s}^{2}\right)$ \\
\hline \hline 28.578 & 16.154 & 352.462 & 199.244 & 0.85 & 21.034 \\
\hline
\end{tabular}

$$
V_{\max }=3.6 \sqrt{R a_{y \max }}=3.6 \times \sqrt{25 \times 21.034}=82.6 \mathrm{Km} / \mathrm{h}
$$

This speed's left turn does not reach or exceed $82.6 \mathrm{Km} / \mathrm{h}$. Once the displacement sensor monitoring the spring displacement is less than $16.154 \mathrm{~mm}$ on the left side or the right side, spring displacement is greater than $199.244 \mathrm{~mm}$, at which the alarm system should be switched on.

\section{CONCLUSION}

1) The method of selection of pressure displacement sensor sensing pressure questioned the measurement of the vertical load of the vehicle being difficult, so on this basis, , the principle of rollover warning based on suspension deformation was proposed.

2) The calculation method of the wheel suspension sprung pressure when the vehicle is turning, and the warning method that takes the lateral load transfer rate as rollover warning threshold, thereby the size of warning threshold of wheel suspension pressure when cornering were determined.

3) Taking a test vehicle for example, rollover warning simulation was carried out in accordance with the early warning methods proposed. The results show that the rollover warning method proposed can predict the state of the vehicle well with its real-time calculation amount being small.

\section{CONFLICT OF INTEREST}

The authors confirm that this article content has no conflict of interest.

\section{ACKNOWLEDGEMENTS}

Research was partially sponsored by the National Natural Science Foundation of China (Grant No.51205151), Project funded by China Postdoctoral Sci-ence Foundation (Grant No. 2013M5312471), Postdoctoral research grants program of Jiangsu Province (Grant No. 1301096C) and The peak of six personnel of Jiangsu Province (Grant No. 2013-ZBZZ020).

\section{REFERENCES}

[1] Z. Zhiguo, "Heavy vehicles rollover warning technology condition and research progress", Communications Science and Technology Heilongjiang, vol. 1, pp. 93-94, 2012.

[2] Z. Zhiguo, and W. Dongdong, "Research status and development trend of side tumbing pre-warning vehicle", Journal of Hebei University of Science and Technology, vol. 34, pp. 108-112, 2013.

[3] L. Jarosi, A. Matteson, and J. Woodrooffe, Trucks involved in fatal accidents fact book 2006. The University of Michigan Transportation Research Institute; Ann Arbor, 2008.

[4] Y. Zhixin, Z. Changfu, and H. Lei, "Algorithms of anti-rollover control for heavy vehicle based on TTR warning", Journal of Jilin University: Engineering and Technology Edition, vol. 39, no. 2, pp. 258-261, 2009.

[5] B. C. Chen, and H. Peng, "Differential-braking-based rollover prevention for sport utility vehicles with human-in-the-loop evaluations", Vehicle System Dynamics, vol. 36, pp. 359-389, 2001.

[6] S. Ming, W. Zuosong, L. Zhigang, Z. Jinhuan, W. Zuo, S. Ning, and $Z$. Meng, "A research on the rollover risk of motor vehicles 
based on simulation analysis", Automotive Engineering, vol. 31, pp. 1173-1176, 2009.

[7] L. Peijun, "Analysis, detection and early warning control of dynamic rollover of heavy freight vehicles", Montreal: School of Mechanical engineering, Concordia University, 1999.

[8] W. Jian, Y. Guizhen, Z. Wei, D. Nenggen, "Real time rollover prediction for vehicle based on principles of sliding mode and fuzzy inference system", Transactions of the Chinese Society for Agriculture Machinery, vol. 41, pp. 1-6, 2010.
[9] W. Aobo, T. Wei, and Z. Yuxian, "Vehicle rollover prevention strategy based on suspension distortion perception", Science \& Technology Information, vol. 26, pp. 70-72, 2013.

[10] L. Zhigang, S. Ming, Z. Jinhuan, H. Jingyao, and M. Chunsheng, "Multi-body dynamics simulations for mini-bus rollover tendency during sharp turns", Journal Tsinghua University Science \& Technology Information, vol. 50, pp. 1286-1289, 2010.

Received: November 14, 2014

Revised: January 6, 2015

Accepted: January 15, 2015

(C) Zhao et al.; Licensee Bentham Open.

This is an open access article licensed under the terms of the Creative Commons Attribution Non-Commercial License (http://creativecommons.org/licenses/by-nc/4.0/) which permits unrestricted, non-commercial use, distribution and reproduction in any medium, provided the work is properly cited. 